\title{
A Tool For ECG Analysis as a Module of a Tele-Monitoring System
}

\author{
http://dx.doi.org/10.3991/ijoe.v12i04.5260
Teresa Rocha ${ }^{1}$, Simão Paredes ${ }^{1}$, Ramona Cabiddu ${ }^{2}$, Ricardo Couceiro ${ }^{3}$, Paulo Carvalho ${ }^{3}$, Jorge Henriques ${ }^{3}$
${ }^{1}$ Instituto Politécnico de Coimbra, Portugal
${ }^{2}$ Federal University of São Carlos, São Carlos, São Paulo, Brazil
${ }^{3}$ Universidade de Coimbra, Coimbra

\begin{abstract}
This work describes a Matlab $^{1}$ tool originally developed in the HeartCycle ${ }^{2}$ European project and updated during the cardioRisk ${ }^{3}$ project. It addresses the analysis of the electrocardiogram (ECG) signal in the context of the management of heart failure (HF) patients.

The toolbox is composed of six modules, focusing on the major clinical aspects relevant to $\mathrm{HF}$ diagnosis: signal delineation, detection of auricular and ventricular arrhythmias, ST segment deviation, and heart rate variability analysis. The effectiveness of the algorithms was validated using private and public datasets.
\end{abstract}

Index Terms-ECG analysis, Matlab simulation tool, heart failure.

\section{INTRODUCTION}

The electrocardiogram (ECG) is a simple, painless and cheap test that registers the heart's electrical activity. As a result it is, definitely, the most common test used to diagnose cardiac diseases [1]. The analysis and the understanding of the ECG, specifically the presence of abnormal rhythms, is decisive in the diagnosis of cardiovascular conditions, and heart failure is not an exception. Although its analysis can be manually performed by professionals, automatic algorithms are of major interest. In fact, they can assist professionals in their decisions, contributing to improve the accuracy of the diagnosis and to considerably reduce the time consuming tasks involved in its analysis.

Several algorithms and methodologies are available in literature addressing the ECG automatic analysis. Moreover, distinct approaches can be used, from basic signal processing techniques to advanced computational intelligence algorithms. Furthermore, different types of elaboration can be performed, namely pre-processing (e.g. noise reduction), delineation (detection of main waves and intervals), alarms and detection of abnormal rhythms.

The main goal of this work is to present the ECG analysis tool (in Matlab) and the respective functionalities that were originally developed during the HeartCycle project [2] particularly applied to the HF condition. The heart rate variability module was improved and updated as a result of the cardioRisk project, specifically in what concerns spectral, non-linear and time domain analysis.

The paper is organized as follows: Section II describes the Matlab analysis tool developed for this purpose, name-

\footnotetext{
Matlab, Mathworks, Inc

www.heartcycle.eu (FP7-ICT-21669)

cardioRisk: PTDC/ EEI-SII/ 2002/2012
}

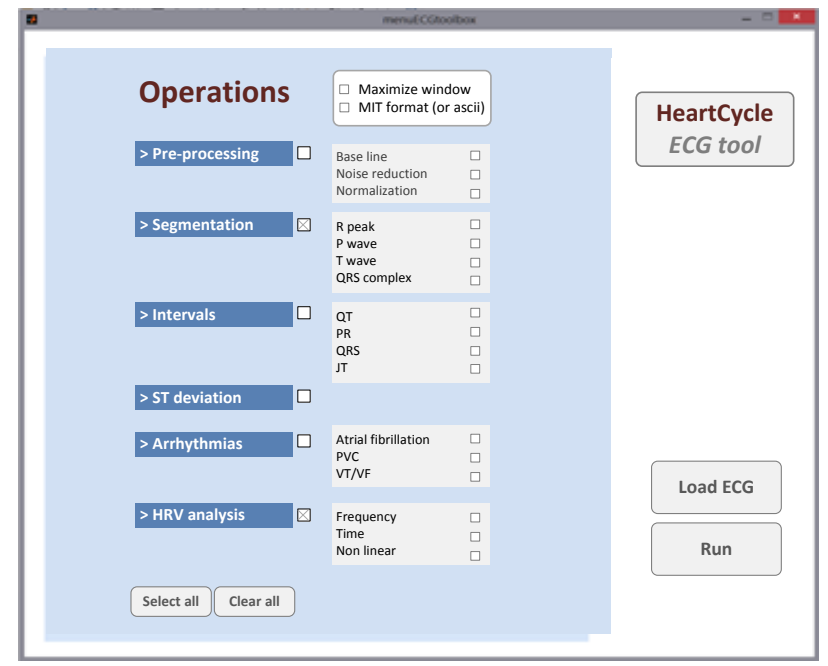

Figure 1. Interface of the Matlab analysis tool.

ly the modules involved. Section III presents the algorithms and the validation results. Section IV shows two illustrative examples and section IV concludes the paper.

\section{MATLAB ANALYSIS TOOL}

Figure 1 depicts the interface of the developed ECG analysis tool.

The tool comprises functionalities to load an ECG (enabling two types of formats) and to perform its analysis. In particular, six main modules are available: i) preprocessing (including noise reduction and base line wandering); ii) segmentation/delineation of the main waves, i.e. P and T waves and QRS complexes; iii) main intervals computation (PR, QT and QRS duration); iv) ST segment shift estimation (used in ischemic events detection); $v$ ) arrhythmias (including atrial fibrillation, premature ventricular contraction, ventricular tachycardia and ventricular fibrillation); vi) heart rate variability analysis (including time, frequency and non-linear parameters). This package and its functionalities were integrated in the decision support system of the HeartCycle tele-monitoring system, aiming the management of heart failure patients.

\section{MATERIAL AND METHODS}

\section{A. Algorithms}

\section{1) Pre-processing}

Among the numerous methods that have been proposed in literature, two particular methods have shown remarkable results, and are especially adapted to deal with noise 
artifacts and capable to operate in an online approach (or near real time approach), which are of major importance in tele-monitoring contexts. These two approaches are based on digital wavelet transforms and multi-scale morphological operators.

As result, in the pre-processing performed: $i$ ) the baseline wander and noise removal was implemented using a wavelet approach; ii) for the segmentation the method proposed by Sun [3] with some adaptations was implemented. In effect, using morphological analysis, the most important fiducial points have been determined, enabling to characterize QRS complex, $\mathrm{P}$ and $\mathrm{T}$ waves, as well as the relevant intervals based on those waves. In particular the following parameters were computed:

- Segmentation: $P$ wave: $\mathrm{P}$ onset, $\mathrm{P}$ peak and $\mathrm{P}$ offset indexes; QRS complex: $\mathrm{Q}$ onset, $\mathrm{Q}$ peak, $\mathrm{R}$ peak, $\mathrm{S}$ peak and $\mathrm{S}$ offset indexes; $T$ wave: $\mathrm{T}$ onset, $\mathrm{T}$ peak and $\mathrm{T}$ offset indexes.

- Intervals: RR, heart rate (bpm), PR interval (s), corrected QT interval (s), Q wave width (s), Q peak height, R peak height, QRS complex duration (s) and corrected JT interval.

\section{2) AF: Atrial fibrillation}

From the clinical perspective the key characteristics of an AF episode is the absence of P waves before the QRS$\mathrm{T}$ complex, which presents a sawtooth like pattern along the cardiac cycle, and the irregularity of the RR intervals. Thus, the proposed strategy [4][5] makes use of these three major physiological characteristics of AF, applied by cardiologists in their daily reasoning: $i$ ) $\mathrm{P}$ wave absence/presence, ii) heart rate irregularity and iii) atrial activity analysis. This knowledge-based approach increases the interpretability of the results to the medical community, while improving detection robustness.

A total of 6 features, $f_{i}, i=1, . ., 6$, have been computed to address the above three characteristics. The features $f_{1}, f_{2}$ and $f_{4}$ are time-domain features; the feature $f_{6}$ is a frequency-domain feature; the $f_{3}$ and $f_{5}$ features are computed using non-linear measures.

- $\mathbf{P}$ wave detection: $\left(f_{1}\right)$, the $\mathrm{P}$ wave presence was assessed by measuring the linear correlation of each $\mathrm{P}$ wave to a normalized $\mathrm{P}$ wave model, created using $\mathrm{P}$ waves extracted from the Physionet QT dataset.

- Heart rate variability: $\left(f_{2}, f_{3}, f_{4}\right)$, in a first phase the RR interval sequence was modelled as a threestate Markov process, being each interval classified as one of the three states (short, regular or long), and characterized by its state transition probability matrix. Three features were computed based on the transition probability, on the entropy of the distribution and on the Kullback-Leibler divergence measure between current window and model distribution.

- Atrial activity analysis: $\left(f_{5}, f_{6}\right)$, the method proposed by [6], was used to cancel the QRS-T components from the ECG signal. Then, the spectrum content of the obtained atrial activity was assessed by computing Kullback-Leibler divergence between each window spectrum and a normalized AF spectrum model derived from the Physionet AF dataset.

\section{3) PVC: Premature Ventricular Contractions}

Most of the algorithms reported in literature for PVC detection are based on features derived from the QRS complex, independently from the surrounding ECG morphological characteristics. However, patients can exhibit several physical, clinical and cardiac conditions, which can affect the ECG morphology in numerous ways. In order to capture patient specific ECG characteristics, for each beat the measurements are compared with those extracted from the neighboring beats. A total of 13 features, $f_{i}, i=1, . ., 13$, have been computed, mainly to characterize the QRS complex shape and its spectral components [7].

- Time domain features: $\left(f_{1}, \ldots, f_{8}\right)$, the first group of features assesses the shape of the QRS complex, $\mathrm{P}$ and $\mathrm{T}$ waves (duration, area, center of mass and amplitude). The RR regularity is also computed.

- Morphological operators: $\left(f_{9}, f_{10}\right)$, two features are based on the ECG signal's morphological derivative, to estimate slops before and after each $\mathrm{R}$ peak.

- Spectral information: $\left(f_{11}, f_{12}, f_{13}\right)$, the last three features are based on the frequency spectrum content, namely the entropy, to assess the concentration of the current spectrum and the Kullback-Leibler divergence between each normalized spectrum and the average of all spectrums.

\section{4) VT: Ventricular tachycardias}

The approach assumes that the fundamental differences in the physiologic origins of normal rhythm and VT, can be discriminated via time ECG shape together with power spectral density analysis. To this aim a total of 11 features $f_{i}, i=1, . ., 11$, have been computed [8].

- Time domain features: $\left(f_{1}, \ldots, f_{6}\right)$, the first feature estimates the morphology of the signal, computing the amount of time that each beat peaks is above or below a given threshold. The second feature basically assesses the heart rhythm. The next four features assessing of small and high derivatives in the ECG signal, enabling to detect abnormal signal amplitudes and slopes.

- Spectral information: $\left(f_{7}, f_{8}, f_{9}\right)$, the energy contained in different frequencies was used as an approach for characterizing the ECG signal. The PSD was evaluated by windowing segments of signal, computed using the Welch's method.

- Non-linear features: $\left(f_{10}, f_{11}\right)$, one feature employs a non-linear transform, in particular the multiplication of backward differences, providing an estimation of extreme variations in the ECG. The other estimates the spatial filling index, computed from the ECG phase space reconstruction diagram.

\section{5) ST deviation}

The ST segment deviation is a measure computed as the difference between the isoelectric point (after the $\mathrm{P}$ wave) and the amplitude of the $J$ point (segment of the ECG that presents a stable behavior, between the end of the QRS complex and beginning of the $\mathrm{T}$ wave). This amplitude, designated as ST segment deviation, is decisive in the assessment of the ischemic condition. The algorithms implemented to evaluate ST segment deviation follow basically two stages [9]. 
1. Baseline removal: Based on $\mathrm{R}$ peaks localization, the entire ECG signal is broken into cardiac cycles using the average of the distances between consecutive $\mathrm{R}$ peaks. Each cardiac cycle is then submitted to a process of baseline removal using Wolf's method [10].

2. J point estimation: The approach for the estimation of ST deviation was based on a time-frequency analysis, in particular using the Wigner-Ville (WV) transform. The basic idea consists in the division of the time frequency map into characteristic areas and, within each specific area, performs the evaluation of particular characteristics. With respect to ST estimation two time bands and one frequency band was considered. Regarding time band, the areas considered were those on the left (isoelectric line) and on the right (ST segment) of the R peak. For each time band it is expect to determine regions where there is no signal activity (the isoelectric point-between the end of $\mathrm{P}$ wave and the begin of QRS complex, and the $J$ pointbetween the end of QRS complex and the begin of $\mathrm{T}$ wave).

Figure 2 depicts this idea, were an electrocardiogram and its corresponding high time-frequency components is shown. Using the minimum of the sum of the high frequency components in each time band, isoelectric and $\mathrm{J}$ points can be obtained, enabling to compute the ST deviation (difference between J point and isoelectric points).

\section{1) HRV: Heart Rate Variability}

Various measures of HRV have been proposed in literature, which can generally be subdivided into time domain, frequency domain and non-linear measures. The algorithms implemented here to determine these measurements follow common approaches found in literature and no special effort was made to derive new measurements. The following parameters are available [11]:

- Time Domain: mean: mean of RR intervals; $S D N N$ standard deviation of RR intervals; SDSD standard deviation of the differences between heart beats (DHB); RMSSD root mean square of the DHB; NN50 number of RR intervals that fall within 50 milliseconds; $p N N 50$ percentage of total NN50.

- Frequency domain: $P S D$ frequency content (Burg and Welch method are available); $p V L$ percentage of very low frequency content $[0-0.04] ; p L F$ percentage of low frequency content $[0.04-0.15] ; p H F$ percentage of high frequency content $[0.15-0.40] ; r L F$ ratio $\mathrm{pLF} / \mathrm{pHF}$.

- Non-linear: sample Entropy $(S E)$, binary Lempel-Ziv Complexity ( $L Z C 1)$, ternary Lempel-Ziv Complexity (LZC2), short term Detrended Fluctuation Analysis (DFA1), long term Detrended Fluctuation Analysis $(D F A 2)$, Fractal Slope $(F S)$, and the two Pointcaré Plots indices (PP1 and PP2).

\section{B. Validation}

\section{1) Classification module}

The accuracy of a classifier is, obviously, highly dependent on the number of classes to be categorized. Clearly, with only two classes each classifier is able to provide a superior classification result, due to the lower complexity of the problem. This fact has justified the design of a distinct classifier (neural network) for each specific task (AF, PVC and VT). The proposed classifiers consist of feedforward neural networks with sigmoidal type activa-

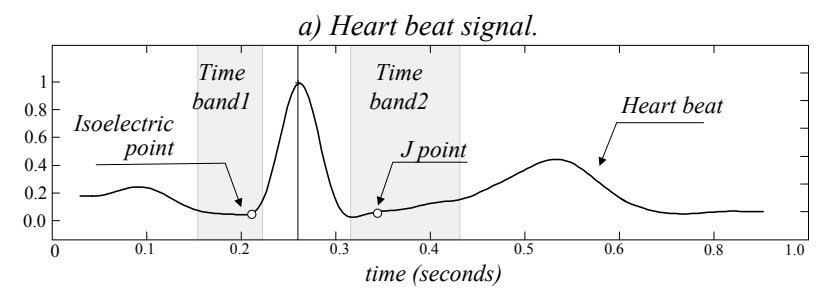

b) High frequency components analysis

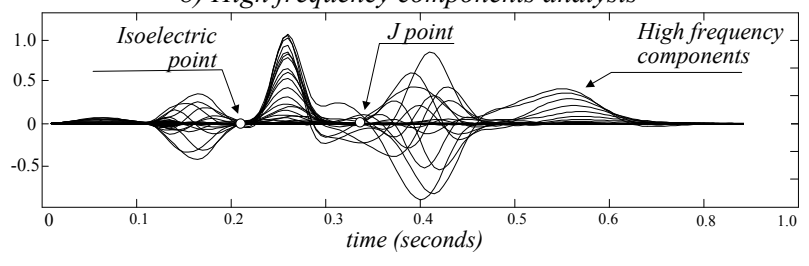

Figure 2. ST segment deviation. a) Electrocardiogram, isoelectric and $\mathrm{J}$ points $b$ ) frequency components (Wigner-Ville transform).

tion functions, being its parameters, i.e., the weights and the bias trained using the Levenberg-Marquardt algorithm.

\section{2) Data sets and performance}

To assess the performance of the algorithms a subset of the Physionet MIT-BIH databases [12] was used, namely AF: atrial fibrillation database (AF); PVC: arrhythmia database; VT: malignant arrhythmia database (VA) and Creighton University ventricular tachyarrhythmia database $(\mathrm{CV})$. For the assessment of the ST deviation the European ST database was used [13]. The results obtained for the ECG segmentation (sensitivity and positive predictive value) and for the arrhythmias detection (sensitivity and specificity) are presented in Table I and Table II, respectively.

The examples hereby presented address the segmentation (delineation) of a common ECG signal, aiming at the identification of its main waves, and the HRV analysis, including time, frequency and non-linear parameters. Figure 3 illustrates the result of a typical ECG segmentation process. It shows a situation where relevant ECG waves were clearly identified ( $\mathrm{P}$ and $\mathrm{T}$ waves, QRS complex and R peak).

\section{ILLUSTRATIVE EXAMPLES}

Figure 4 illustrates some of the parameters computed from the HRV, using the intervals variations between consecutive heart beats as inputs. The analysis tool addresses typical HRV analysis parameters, which reveal important information from the clinical perspective. These are: time domain (mean, SDNN-standard deviation of normal intervals), frequency domain components (very low, low and high frequency components), and non-linear parameters (Poincare plots, complexity and entropy).

TABLE I. SEGMENTATION - DETECTION PERFORMANCE

\begin{tabular}{cccc|ccc}
\multicolumn{4}{c|}{ Sensitivity [\%] } & \multicolumn{3}{c}{ PPV [\%] } \\
\hline \hline Wave & P & QRS & T & P & QRS & T \\
Mean & 93.17 & 99.80 & 97.64 & 96.82 & 99.70 & 98.00 \\
\hline \hline
\end{tabular}

TABLE II. ARRHYTHMIAS - DETECTION PERFORMANCE

\begin{tabular}{ccccc} 
& AF & PVC & VT (VA) & VT (CV) \\
\hline Sensitivity & 92.2 & 96.3 & 90.7 & 91.8 \\
Specificity & 91.4 & 99.1 & 95.0 & 96.9 \\
\hline \hline
\end{tabular}




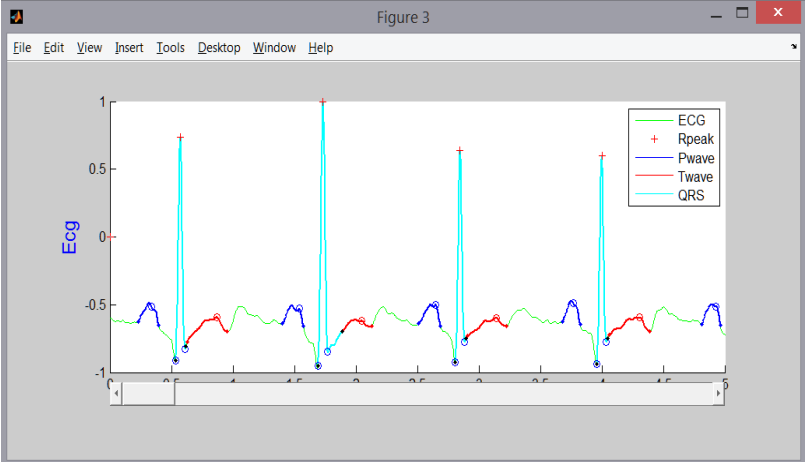

Figure 3. ECG typical segmentation.

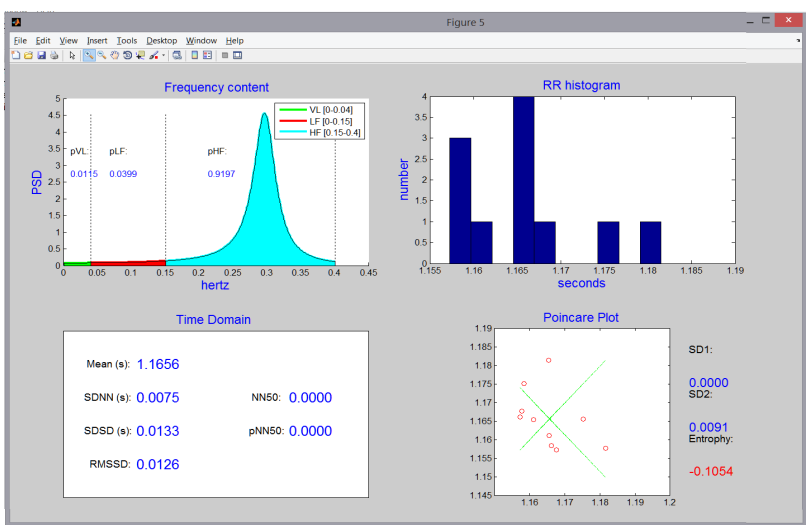

Figure 4. HRV analysis.

\section{CONCLUSIONS}

A Matlab analysis tool was briefly presented in this paper, which was originally implemented during the HeartCycle European project and improved in the cardioRisk project. This package is modular, presenting a solution for the analysis of the ECG, enabling to compute some significant parameters, as well as to detect normal and abnormal events, typically used by physicians to support their decisions when dealing with HF patients.

The validation of the algorithms was based on public databases. Classification results show that the proposed approach can be used to discriminate between different types of arrhythmia with state of the art accuracy.

\section{REFERENCES}

[1] McMurray et al (2012); ESC Guidelines for the diagnosis and treatment of acute and chronic heart failure 2012; European Heart Journal, 33, 1787-1847.

[2] Reiter, H. and N. Maglaveras; "HeartCycle: compliance and effectiveness in HF and CAD closed-loop management"; Conf. Proc IEEE Eng Med Biol Society, 299-302, 2009. http://dx.doi.org/10.1109/iembs.2009.5333151

[3] Sun, Y., K. Chan, S. Krishnan; "Characteristic wave detection in ECG signal using morphological transform"; BMC Cardiovascular Disorders 5:28, 2005. http://dx.doi.org/10.1186/1471-2261-5-28

[4] Couceiro, R., P. Carvalho, J. Henriques, M. Antunes; "Detection of Atrial Fibrillation Using Model-based ECG Analysis"; ICPR-
2008; Tampa, USA, August 20-24, 2008. http://dx.doi.org/10.1109/icpr.2008.4761755

[5] Carvalho, P., J. Henriques, R. Couceiro, M. Harris, M. Antunes, J. Habetha; Model-Based Atrial Fibrillation Detection; Chapter 5 ECG Signal Processing, Classification and Interpretation; Ed: Gacek and Pedrycz; 99-133, ISBN:978-0-85729-867-6, 2012.

[6] Sanchez, C., J. Millet, J. Rieta, F. Castells, J. Ródenas, V. Ruiz; "Packet Wavelet Decomposition: An Approach for Atrial Activity Extraction; IEEE Computers in Cardiology", 29:33-36, 2002. http://dx.doi.org/10.1109/CIC.2002.1166700

[7] Couceiro, R., P. Carvalho, J. Henriques, M. Antunes; "On the detection of premature ventricular contractions"; EMBC-08, Vancouver, Canada, August 20-24, 2008. http://dx.doi.org/10.1109/ iembs.2008.4649349

[8] Henriques, J., P. Carvalho, M. Harris, M. Antunes, R. Couceiro, M. Brito, R. Schmidt; "Assessment of Arrhythmias for Heart Failure Management"; phealth2008 - International Workshop on Wearable Micro and Nanosystems for Personalised Health; Valencia, Spain, May 21-23, 2008.

[9] Rocha, T., S. Paredes, P. Carvalho, J. Henriques, M. Harris, J. Morais, M. Antunes; "A lead dependent ischemic episodes detection strategy using hermite functions"; Biomedical Signal Processing Control 5, 271-28, 2010. http://dx.doi.org/10.1016/ j.bspc.2010.07.001

[10] Wolf, A. "Automatic Analysis of Electrocardiogram Signals using Neural networks", PUC-Rio, Ms. Thesis, n 0210429/CA2004.

[11] Cabiddu, R., S. Mariani, J. Henriques, S. Cerutti, A. Bianchi; "Non-linear Indices of Heart Rate Variability in Heart Failure Patients during Sleep"; XIII Mediterranean Conf. MBEC, IFMBE Proceedings 41, 690-693, Seville, Spain, September, 25-28, 2013.

[12] Physionet; http://www.physionet.org/physiobank/database.

[13] Taddei, A. et al, "The European ST Database: standard for evaluating systems for the analysis of ST-T changes in ambulatory electrocardiography “, Eur. Heart J., 13, 1164-72, 1992.

\section{AUTHORS}

T. Rocha is with Instituto Politécnico de Coimbra, DEIS, Quinta da Nora, 3030-199 Coimbra, Portugal (email: teresa@isec.pt).

S. Paredes is with Instituto Politécnico de Coimbra, DEIS, Quinta da Nora, 3030-199 Coimbra, Portugal (email: sparedes@isec.pt).

R. Cabiddu is with the Cardiopulmonary Physiotherapy Laboratory, Federal University of São Carlos, Rodovia Washington Luís, Km 235, Jardim Guanabara, 13565-905, São Carlos, São Paulo, Brazil (e-mail: ramona.cabiddu@gmail.com).

R. Couceiro is with CISUC, Departamento de Engenharia Informática, Universidade de Coimbra, 3030-290, Coimbra, Portugal (email: rcoucer@dei.uc.pt).

P. Carvalho is with CISUC, Departamento de Engenharia Informática, Universidade de Coimbra, 3030-290, Coimbra, Portugal (email: carvalho@dei.uc.pt).

J. Henriques is with CISUC, Departamento de Engenharia Informática, Universidade de Coimbra, 3030-290, Coimbra, Portugal (email: jh@dei.uc.pt).

This work was supported by HeartCycle (FP7/ICT/21669), CardioRisk (PTDC/EEI-SII/2002/2012) and H2020 LINK/692023. Submitted 13 November 2015. Published as resubmitted by the authors 24 January 2016. 